\title{
Generation of a 3D Printed Brain Model from MRI Scan Data to Assist in Brain Surgery using Open Source Tools
}

\author{
Neeraj Kulkarni \\ Department of Mechanical Engineering, \\ DBIT, University of Mumbai, \\ Mumbai, Maharashtra, India \\ neeraj.kulkarni@gmail.com \\ Prof. Shreeprasad Manohar \\ Department of Mechanical Engineering, \\ DBIT, University of Mumbai, \\ Mumbai, Maharashtra, India
}

\author{
Siddhi Patil \\ Department of Mechanical Engineering, \\ DBIT, University of Mumbai, \\ Mumbai, Maharashtra, India
}

\author{
Arunkumar Kashyap \\ Department of Mechanical Engineering, \\ DBIT, University of Mumbai, \\ Mumbai, Maharashtra, India
}

\begin{abstract}
The potential of medical 3D printing for improved patient treatment attained recognition after the MRI (Magnetic Resonance Imaging) technology got invented almost 30 years ago. Further development in this technology focused on providing enhanced quality images, speed and patient comfort. This project work aims to convert the MRI scan data of brain into its equivalent 3D Model using open source tools, which provide the facility of improved processing, and this in turn, aids in converting the same into a sliced model and then 3D Prints it using Fused Deposition Modeling technique. This helps to generate physical, patient-specific 3D models of the brain. The benefits of this work include planning complex surgical interventions in the pre-operative stage thus, reducing the steps involved in implant/removal as well as the time span for which the patient is kept under the impact of anesthesia. This also has an additional advantage of helping with intraoperative orientation. It is understood that, this model will prove to be highly useful with respect to treating a complex organ like the human brain, since its intricate shape makes its $3 D$ rendering a difficult task. Thus, the creation of a live size model of the patient's brain with cut-sections at the tumor's location will definitely assist the neurosurgeons with brain surgeries involving tumor removal.
\end{abstract}

Keywords- Magnetic Resonance Imaging (MRI), Medical 3D Printing, Fused Deposition Modeling, Intraoperative orientation, Brain surgeries.

\section{INTRODUCTION}

Surgery is the most common treatment for brain tumor. Brain surgeries are very critical as even a slightest miscalculation may cause an irreparable damage to the patient. Thus, a 3D printed life-size patient specific cut-section model of a brain (with tumor) will help the surgeon to diagnose the exact location of the tumor much more effectively. Thus, he/she can pre-plan the surgery more efficiently. The cutsections of this model will junction at the tumor and will be made as per the surgeon's demand. To generate a patient specific cut-section 3D printed model of a brain having tumor, first, the surgeon provides MRI scan of patient's brain to the team of engineers who will use it to design the 3D model using various $\mathrm{CAD}$ techniques and then obtain its $3 \mathrm{D}$ print using specific additive manufacturing technologies. The model is then shipped back to the surgeon.

\section{A. MRI Scan}

Magnetic Resonance Imaging or MRI uses strong magnetic fields that interact with human body to produce detailed images of the insides of the body. It can capture the detailed structure brain and its surrounding components along with all the normal and abnormal tissues within. It is a painless, safe and extremely accurate method for detection of swelling, bleeding, tumors in the brain. MRI scan uses DICOM (Digital Imaging and Communications in Medicine) technique, which is both a communication protocol and a file format which can store medical information, such as ultrasound and MRI images, along with the patient's information, all in one file.

\section{B. Additive Manufacturing}

Additive manufacturing (AM) is a technology which builds a 3D object layer-by-layer using a 3D printer. The 3D model is designed using CAD techniques and saved in digital formats like STL or its versions. This digital data is then transferred to the $3 \mathrm{D}$ printer. The $3 \mathrm{D}$ printer then starts printing the base layer by moving a nozzle that ejects molten material over the build plate. The build plate the moves down by specified layer height and next layer in built on top of base layer and so on. Each successive layer bonds to the preceding layer of molten or semi-molten material. As the material cools these layers fuse together to form a 3D object. The materials used can be nylon, thermoplastics, ceramics, composites etc.

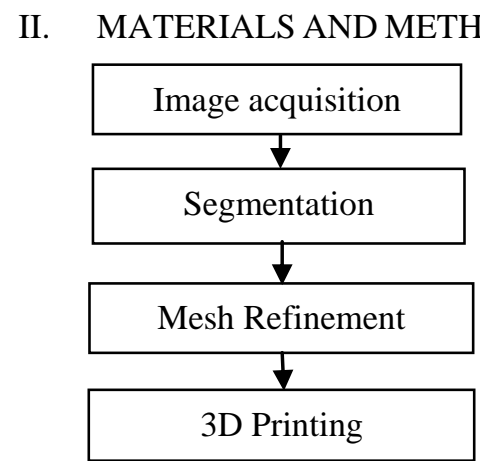


Fig. 1. Flow chart depicting the process of obtaining 3D models from medical images [ $\underline{3}]$.

\section{A. Image acquisition}

The MRI scan of patient's head is required. The MRI scan shows the most accurate 2D images of the patient's brain and the inside region as well as the surrounding organs, tissues, glands, etc. Any anomalies like tumors, clots and internal injuries are visible. The MRI scan is a group of various images each showing a different cut-section of patient's head. There are 3 groups of images depict cut-sections from top to bottom, front to back and left to right. These images are in grey scale.

\section{B. Segmentation}

The aim is to obtain only the 3D model of the patient's brain and the tumor located in it excluding everything else. Thus, brain and tumor tissues must be isolated from the other components in the image. This process is called segmentation. Within the medical images each tissue has a characteristic range of pixel intensities [3] . Segmentation techniques exploit this fact for the purpose of digitally isolating the desired tissue for performing individual operations on it. Here, the software used is an open source software called 3D slicer. Other such software's are Seg3D and FreeSurfer.

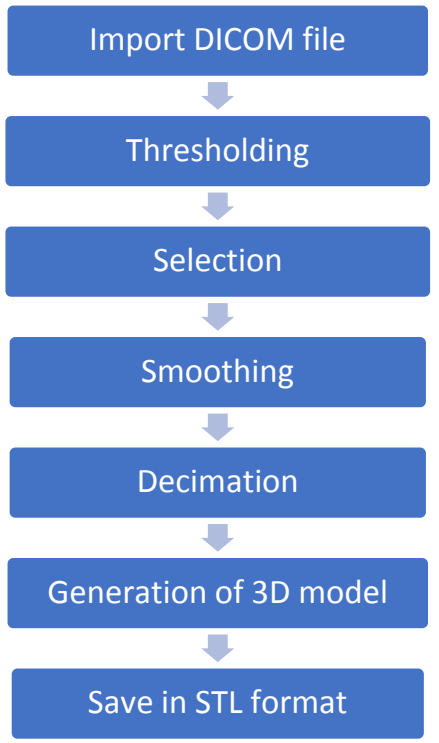

Fig. 2. Flow chart of complete segmentation process

\section{- Import DICOM file}

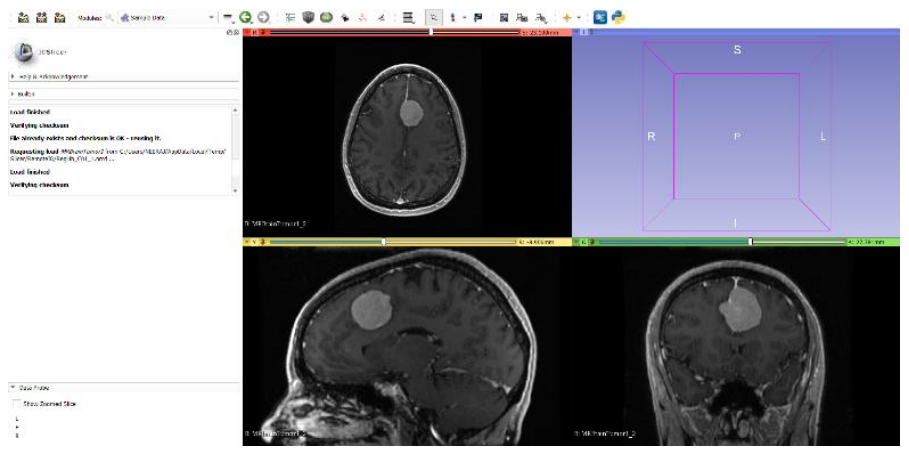

Fig. 3. 3D slicer user interface after loading the DICOM data.

\section{- $\quad$ Thresholding}

Different tissues have different density and biological structure hence during MRI scan they interact differently with magnetic field. This results in each tissue having different range of pixel intensity in the images. Thresholding tool is used for segmenting the desired organ or tissue from the medical images by classifying the pixel range of that organ or tissue. By identifying the pixel range of brain and tumor tissues, we can perform thresholding operation and segment them from rest of the tissues and skull present in the image.

\section{- $\quad$ Selection}

In each consecutive image slice, the areas of tumor and brain tissues are selected in turn. This selection is visualized via a pre-defined color. Due to thresholding operation performed previously, every detail of the brain and tumor tissue geometry is covered with minimum to no interference.

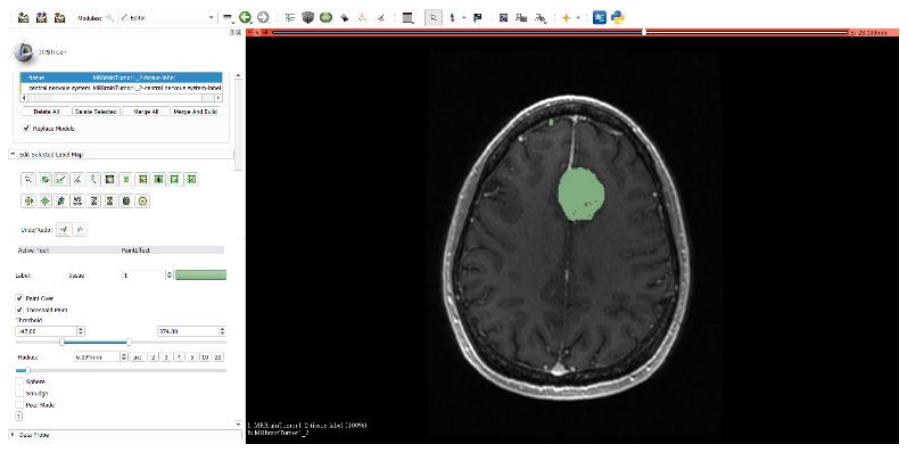

Fig. 4. : Painting the tumor in each image in the red slice one at a time.

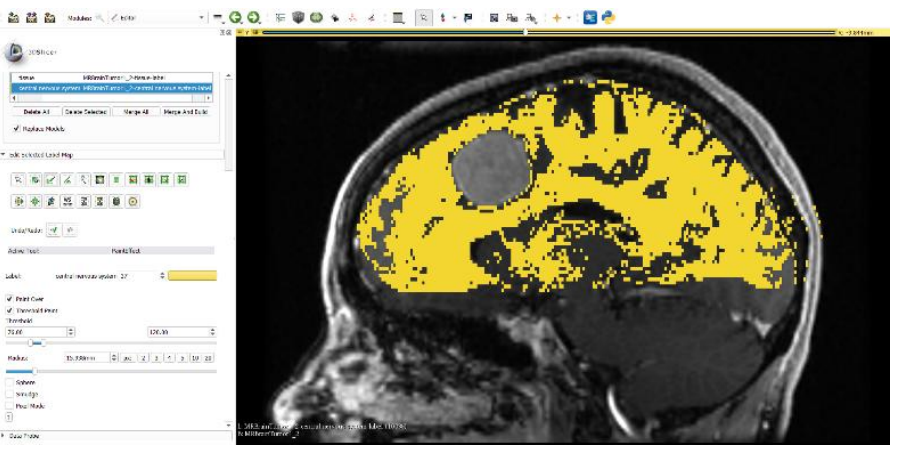

Fig. 5. Painting the brain in each image in yellow slice one at a time.

\section{- $\quad$ Smoothing}

This operation is used to increase the smoothness of the surface of the 3D object. A smooth object has a continuous surface and its surface polygons connect smoothly to their neighboring polygons. There are two smoothing filters namely Laplacian and Sinc. Laplacian filter's algorithm performs smoothing in specified number of iterations. Sinc filter's algorithm performs smoothing by approximation of surface polynomials by specified degree. []

\section{- Decimation}

This operation performs a series of local operations on a mesh to gradually reduce the number of triangles and vertices 
until the desired reduction is achieved or no more reductions are possible due to topological or other constraints [8].

\section{- Generation of 3D model}

$3 \mathrm{D}$ model is generated by stacking the selected areas in all the consecutive image slices. Smoothing and decimation operations give the model real life geometry to a certain degree.

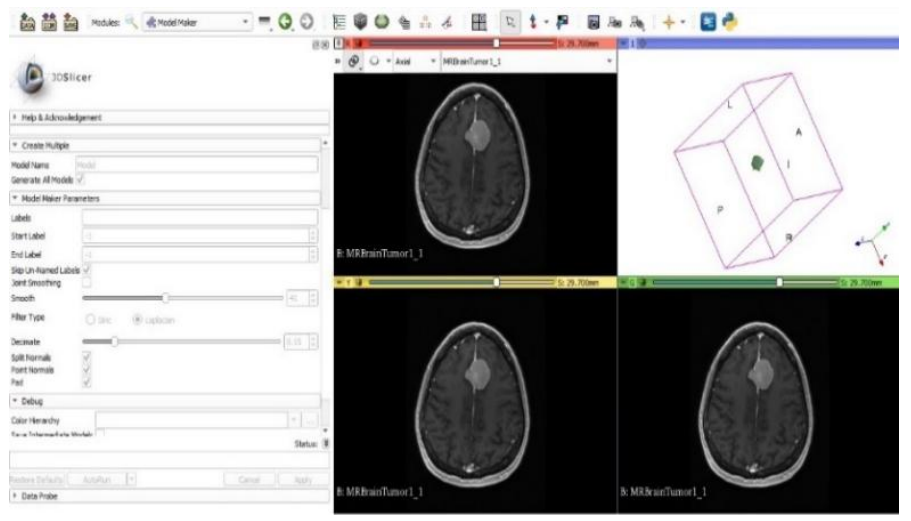

Fig. 6. 3D model of tumor generated by combining all the colored layers in the images in the blue slice called Tabbed 3D.

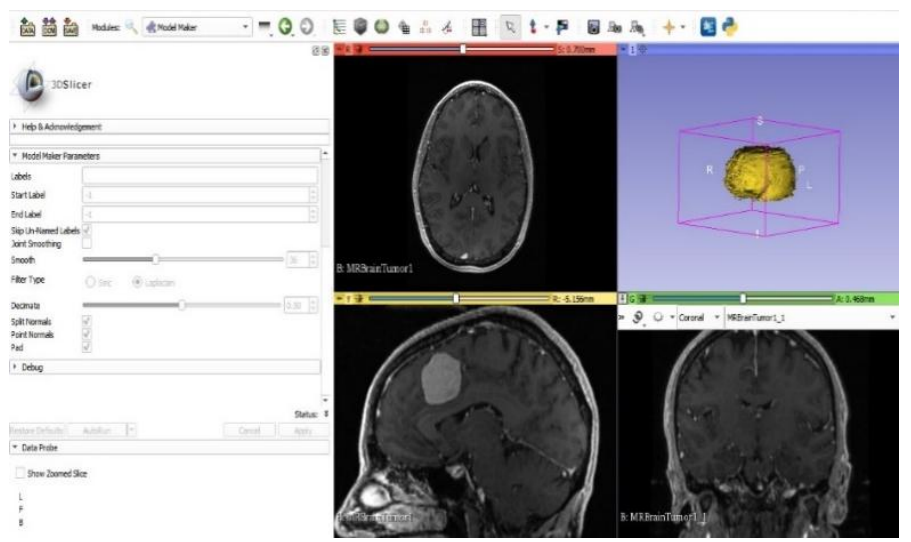

Fig. 7. 3D model of brain generated in the Tabbed 3D by combining the colored layers in each image.

\section{- Save in STL format}

STL (Standard Tessellation Language) is a common file format for digital 3D data. The 3D data is stored in form of a triangular mesh.

\section{Mesh Refinement}

The 3D model obtained for segmentation is not fit for use. It has several defects, discontinuities and is not watertight therefore, we need to do mesh refinement. There are three purposes of mesh refinement. First is repairing, in which all the errors and discontinuities arising during the segmentation process, are removed. Second is smoothing, in which the staircasing errors generated as a result of the resolution of the medical image are removed. Third is appending, in which the unwanted parts which were generated during the segmentation process, are removed and other useful structures are combined to make it usable [3]. The software used here is Meshmixer which is an open source software by Autodesk.

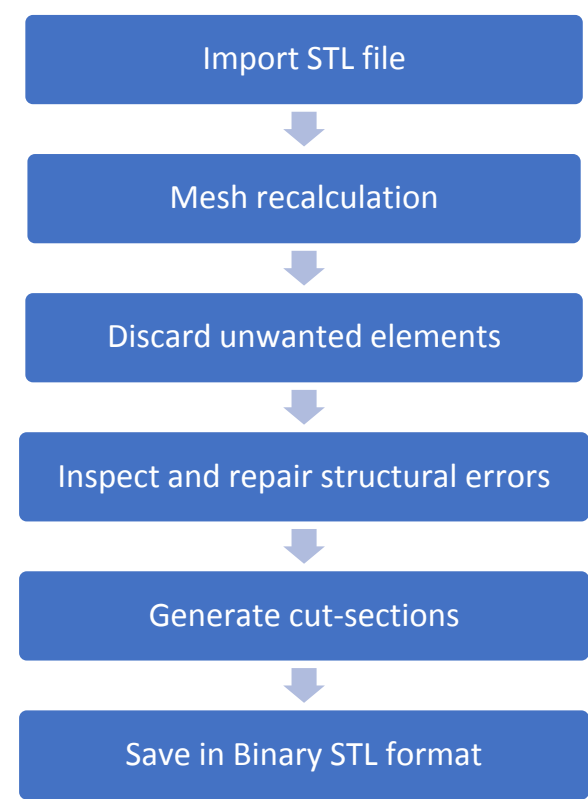

Fig. 8. Flow chart of complete showing mesh refining process.

- $\quad$ Import STL File

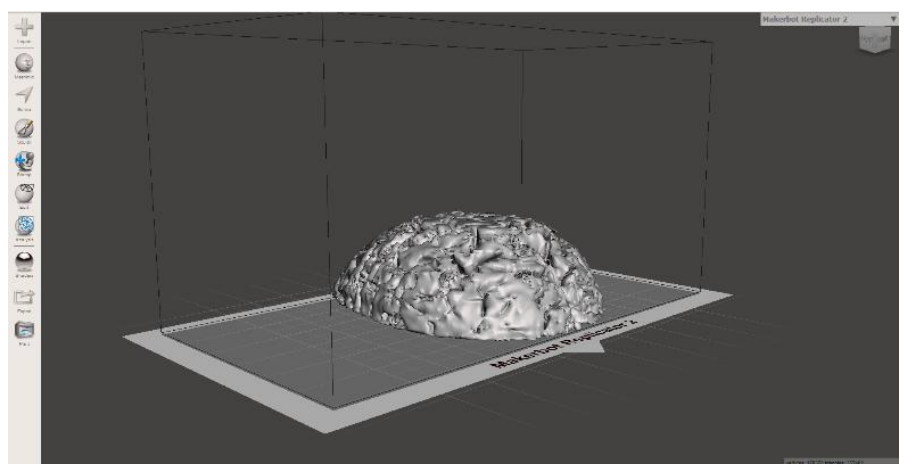

Fig. 9. STL file of brain opened in Meshmixer interface.

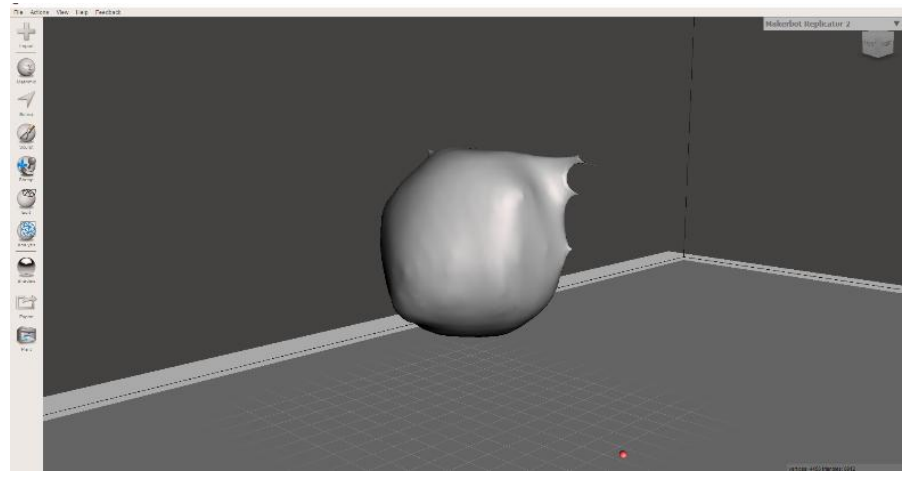

Fig. 10. STL file of tumor in the Meshmixer interface.

\section{- Mesh Recalculation}

This results in creation of blocky or low poly versions of the present model. Meshmixer provides Make Solid tool for this task. It will completely recalculate all triangles in the model, problematic or not. This continuously repairs all problems that the model may undergo, but it degrades the 
quality of the model and increases the file size by quite a margin.

There are different types of solids which the model can be converted into as per required:

1. Fast - This mode helps in meshing the voxel approximation, and then re-projecting the vertices back onto the input surface. This is quite fast and works well in simple cases.

2. Blocky - This mode simply meshes the voxel cubes, giving a blocky object.

3. Accurate - This mode is used when a complete round off model with smooth edges is desired.

4. Sharp Edge Preserving - This mode is used if sharp edges need to be preserved. [9]

Other variable parameters which play a defining role in obtaining satisfactory structure and appearance for a 3D model are:

1. Solid Accuracy- Sets the number of calculations done to the resulting mesh to match it to the shape of the original mesh.

2. Mesh Density- Specifies the number of times to divide your current mesh faces so that a smoother curvature can be achieved. More the mesh density more the filesize and time required for 3D printing the model.

3. Offset Distance- will offset the resulting mesh by a set distance from the original mesh. Positive numbers offset out from the mesh surface, and negative numbers offset the mesh in.

4. Minimum Thickness- Increases the thickness of structures like columns and narrow walls within the model to a set minimum thickness. This option can be used to make all details in the model visible.

5. Cull Edges Threshold- Reduces the mesh before applying the any calculations. It gives unpredictable results. [9]

\section{- $\quad$ Discard unwanted elements}

Segmentation process is not hundred percent accurate. Therefore, a number of very small disconnected 3D particles are generated which appear floating around the main body of brain and tumour. These must be deleted or else they will generate dysconnectivity errors.

\section{- Inspect and repair structural errors}

For an intricate structure like brain, there may be a number of structural and geometric errors within the model. Meshmixer's Inspector algorithm points these errors by three different coloured markers:

1. Blue Markers - If the model contains holes, a blue marker will point at it. From a dropdown menu on the left side, the user can select one of the three different hole-filling methods, namely; Minimal Fill, Flat Fill and Smooth Fill. The user may have to experiment with them. Different methods will work better with different models.
2. Magenta Markers - Magenta markers point at disconnected or very small parts of the model, these will simply be deleted.

3. Red Markers - Red markers show "non-manifold" geometry that cannot exist in the real world. This certainly poses a problem if a 3D print of the model is desired. Meshmixer tries to fix the problem if any of the markers is clicked. However, if the marker turns black, this indicates that the intended function did not succeed and the problem continues to persist.

Meshmixer also provides an option to automatically repair all of these problems together. However, this option comes with a drawback that there is good chance of useful information getting manipulated or deleted.

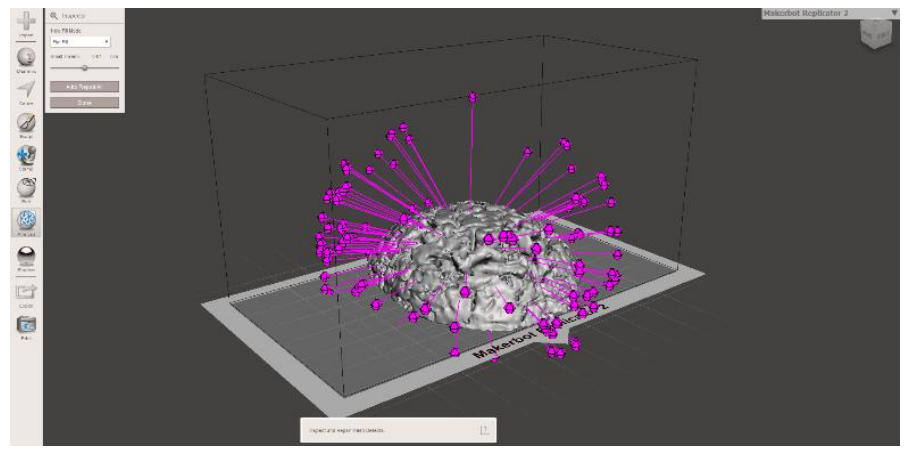

Fig. 11. Errors or defects in structure indicated by pointers.

\section{- Generate Cut-Sections}

Slice the model at required planes to obtain cut-sections. The Plane Cutting tool in Meshmixer gives the liberty to choose the position and angle of cutting plane with respect to the object.

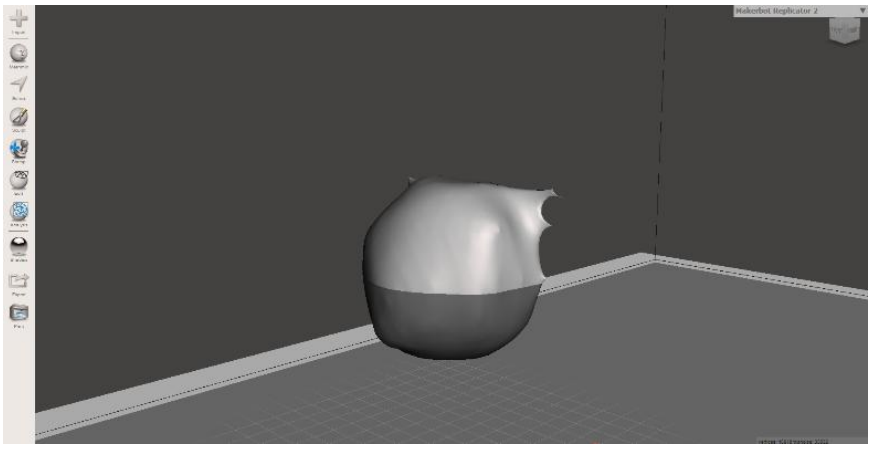

Fig. 12. Tumor divided in two cut-section from the center.

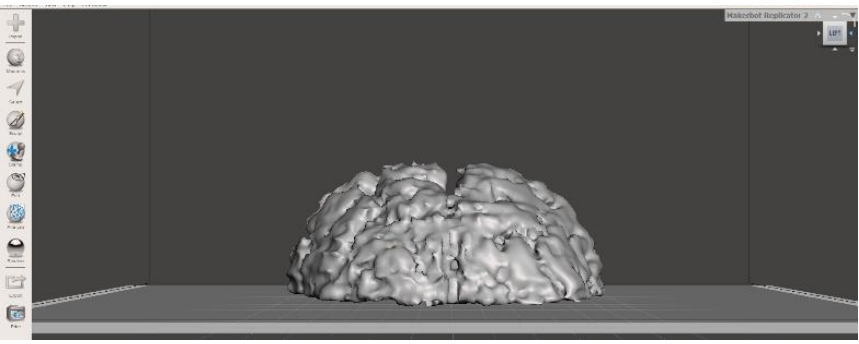

Fig. 13. Front cut-section of brain. 


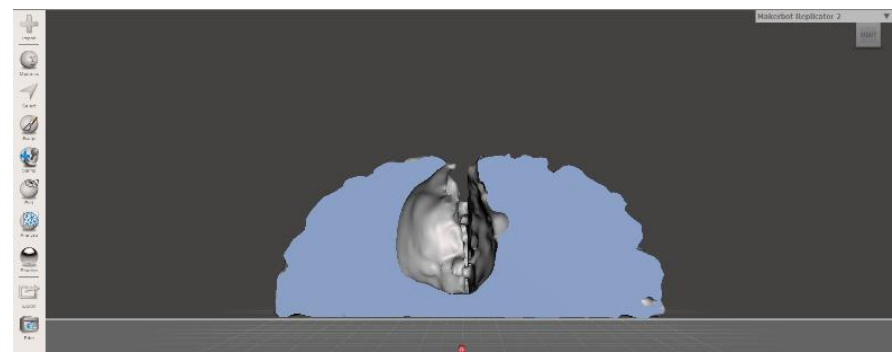

Fig. 14. Front cut-section of brain from behind, cavity represents the volume occupied by tumor.

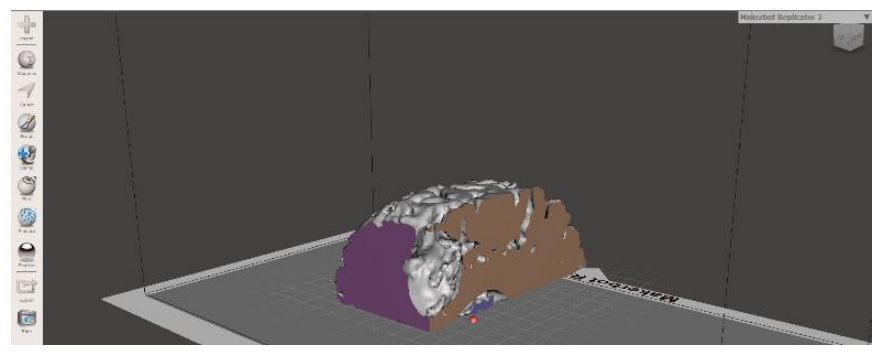

Fig. 15. Back (left side) cut-section of brain.

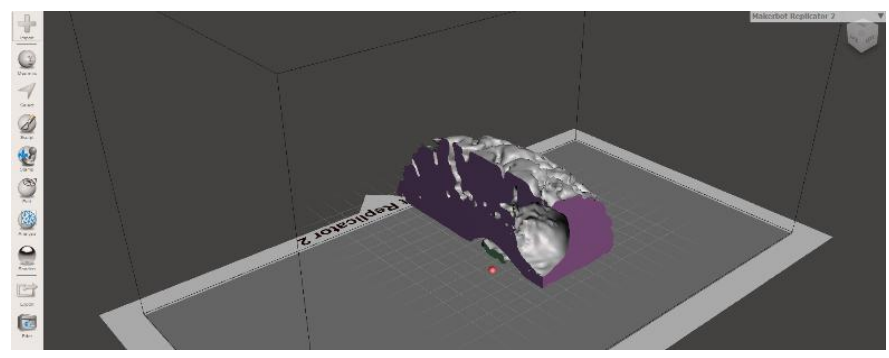

Fig. 16. Back (right side) cut-section of brain.

\section{- Save in Binary STL format}

The binary representation of the STL file format is a simple, openly documented format for describing the surface of an object as a triangular mesh. STL has become a de facto standard for rapid prototyping and 3D printing. For practical use, since its files are more compact, the binary variant is more commonly used for 3D printing.

\section{D. $3 D$ Printing}

The final step in the process involves bringing the model to life by using the $3 \mathrm{D}$ printing technique. The $3 \mathrm{D}$ printer used here is 'Ultimaker 3 Extended' and the printing material is nylon. The 3D printer cannot directly use the binary STL file. The model needs to be sliced and parameters such as orientation of model on build plate, printing speed and layer height. This is done using an open source software called Ultimaker Cura. The file generated by Ultimaker Cura contains all the information needed for 3D printing which is then loaded in the $3 \mathrm{D}$ printer for completing the task.

\section{RESULTS AND DISCUSSION}

The model thus designed, consists of the brain and the tumor only at a ratio of $1: 1$. The tumor is printed in two pieces, which are later joined together using adhesive. There are three cut-sections of the brain that form a junction at the location of the tumor. The cut-sections of the brain form a cavity when joined at their junction where the tumor fits. The tumor can also be painted with a different color like red, to make the model color coded. The design gives the exact location of tumor in the brain from top, left, right, front and back views thus achieving its purpose.

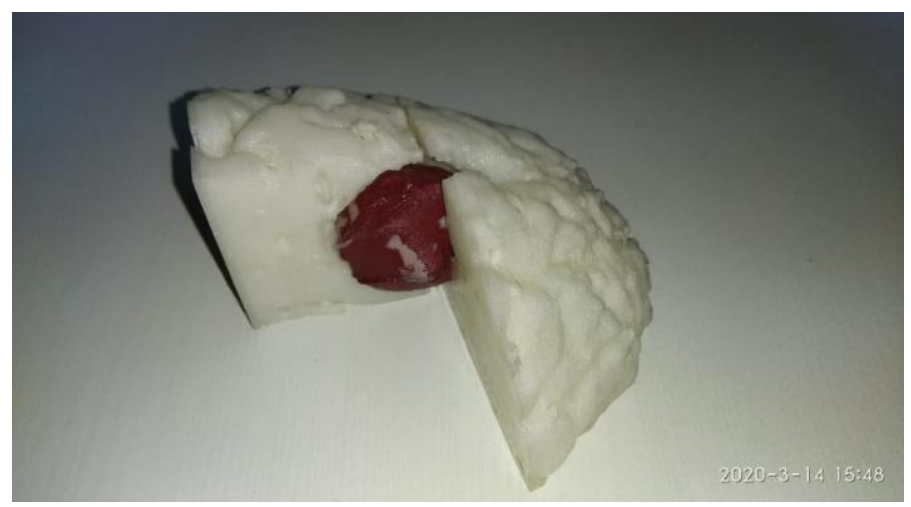

Fig. 17. Final 3D printed cut-section patient specific brain-tumor model

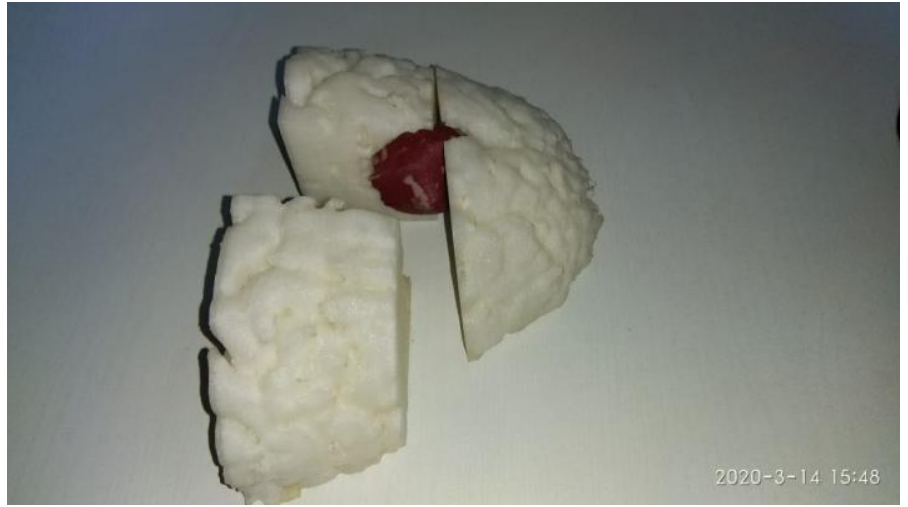

Fig. 18. Tumor exactly fits in the cavity at the junction of the cut-section giving its exact location from various angles.

The importance of the above model was discussed with a practicing neurosurgeon. The discussion can be summarized in the following points-

- The model should be of 1:1 scale.

- There should be as many cut-sections as possible leading to the tumor.

- If possible, it should be made feasible to incorporate other elements like scalp, skull, nerves and blood vessels in the model as per the surgeon's demand in certain specific parts of the model or in the whole model. Each component being detachable and having individual cut-sections.

- The model should have different colors for each component i.e. it should be a color-coded model.

- The model should incorporate as much detail as possible.

\section{CONCLUSION}

This method is shown to generate appropriate result and give an exact cut-section 3D model of the human brain approved by doctors. This 3D model will be helpful before the 
surgery for planning and practice purposes as well as while performing the surgery. 3D models have the cut-sections that junction at the tumour. More cut-sections may be added as per surgeon's demand. This model shows the exact location of the tumour in the brain, so that the doctor can decide the path for surgery which in turn will be well-organized and accurate.

\section{FUTURE SCOPES}

With the advancement in this method, doctors can obtain the exact brain size of the patients possessing- varying brain density and tumour size. This proves to be very beneficial for the doctors who are required to perform the surgery as they can feel the tumour inside the brain via needles. Hence, the chances of success of the operation increase manifold. This method/model is aimed at bringing about revolutionary changes in the field of medical science and brain surgery, considering that the process of $3 \mathrm{D}$ printing is highly reliable, cheap as well as widely used.

\section{ACKNOWLEDGEMENTS}

We would like to thank our guide Prof. Shreeprasad Manohar and the entire Department of Mechanical Engineering of Don Bosco Institute of Technology, Kurla for their technical assistance. We would also like to especially mention and thank Dr. Hitendra Dahivadkar, neurosurgeon, The Kalyan Hospital for giving his time and giving us his valuable advice.

\section{REFERENCES}

[1] Haleem A, Javaid M, Role of CT and MRI in the design and development of orthopaedic model using additive manufacturing,
Journal of Clinical Orthopaedics and Trauma (2018), doi: 10.1016/j.jcot.2018.07.002.

[2] Herrmann $\mathrm{K}-\mathrm{H}$, et al. 3D printing of MRI compatible components: Why every MRI research group should have a low-budget 3D printer. Med Eng Phys (2014), http://dx.doi.org/10.1016/j.medengphy.2014.06.008

[3] BuËcking TM, Hill ER, Robertson JL, Maneas E, Plumb AA, Nikitichev DI (2017) From medical imaging data to 3D printed anatomical models. $\begin{array}{lll}\text { PLoS ONE 12(5): } & \text { e0178540. }\end{array}$ https://doi.org/10.1371/journal.pone.0178540

[4] Andrew Squelch, PhD, 3D Printing and Medical Imaging, Journal of Medical Radiation Sciences, doi: 10.1002/jmrs.300

[5] F. Rengier, A. Mehndiratta, H. von Tengg-Kobligk, C. M. Zechmann, R. Unterhinninghofen, H.-U. Kauczor, F. L. Giesel, 3D printing based on imaging data: review of medical applications, International Journal of Computer Assisted Radiology and Surgery, DOI 10.1007/s11548-0100476-x

[6] Ghazanfar Latif, M. Deriche, Arfan Jaffar, Anwar M. Mirza, Brain MRI Segmentation and 3D Reconstruction, Conference on Computer Science \& Computational Mathematics, 2012.

[7] Wake N, Chandarana H, Huang WC, Taneja SS, Rosenkrantz AB. Application of anatomically accurate, patient-specific 3D printed models from MRI data in urological oncology. Clinical Radiology. 2016; 71 (6): 610-614.

[8] 3D Slicer Documentation [internet], Available: https://www.slicer.org/wiki/Documentation/4.2/Modules/ModelMaker

[9] Digital Media Tutorial Written by Trevor Williams, Basic Model Preparation with Meshmixer [internet], Available: https://digitalmedia.architecture.yale.edu/sites/default/files/files/Meshmi xer.pdf

[10] Autodesk Meshmixer [Internet]. [cited 1 Jan 2016]. Available: http://www.meshmixer.com/ 